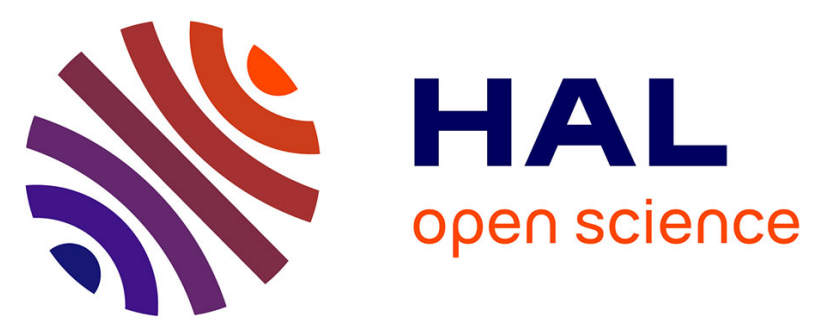

\title{
ARX model for interstitial glucose prediction during and after physical activities
}

\author{
Hector M. Romero-Ugalde, Mael Garnotel, M. Doron, P. Jallon, G.
} Charpentier, S. Franc, E. Huneker, Chantal Simon, S. Bonnet

\section{To cite this version:}

Hector M. Romero-Ugalde, Mael Garnotel, M. Doron, P. Jallon, G. Charpentier, et al.. ARX model for interstitial glucose prediction during and after physical activities. Control Engineering Practice, 2019, 90, pp.321-330. 10.1016/j.conengprac.2019.07.013 . hal-02369251

\section{HAL Id: hal-02369251 \\ https://hal.science/hal-02369251}

Submitted on 25 Oct 2021

HAL is a multi-disciplinary open access archive for the deposit and dissemination of scientific research documents, whether they are published or not. The documents may come from teaching and research institutions in France or abroad, or from public or private research centers.
L'archive ouverte pluridisciplinaire HAL, est destinée au dépôt et à la diffusion de documents scientifiques de niveau recherche, publiés ou non, émanant des établissements d'enseignement et de recherche français ou étrangers, des laboratoires publics ou privés.

\section{다)(1) $(5$}

Distributed under a Creative Commons Attribution - NonCommercial| 4.0 International 


\footnotetext{
${ }^{*}$ Corresponding author

Email address: hector.m.romero.ugalde@gmail.com (Hector M. Romero-Ugalde) Preprint submitted to Control Engineering Practice

June 14, 2019
}

\section{ARX model for interstitial glucose prediction during and after physical activities}

\author{
Hector M. Romero-Ugalde, ${ }^{\mathrm{a}, \mathrm{d}, *}$, M. Garnotel ${ }^{\mathrm{b}}$, M. Doron ${ }^{\mathrm{a}}$, P. Jallon ${ }^{\mathrm{a}}, \mathrm{G}$. \\ Charpentier $^{\mathrm{c}}$, S. Franc ${ }^{\mathrm{c}}$, E. Huneker ${ }^{\mathrm{d}}$, C. Simon ${ }^{\mathrm{b}}$, S. Bonnet ${ }^{\mathrm{a}}$ \\ ${ }^{a}$ Univ. Grenoble Alpes, F-38000 Grenoble France. CEA, LETI, MINATEC Campus, \\ F-38054 Grenoble, France. \\ ${ }^{b}$ CARMEN INSERM U1060/Université de Lyon 1/INRA U1235, CRNH-Rhône-Alpes, \\ Lyon, France. \\ ${ }^{c}$ Centre Hospitalier Sud-Francilien, Department of Diabetes and Endocrinology, \\ Corbeil-Essones, France and with Centre d'Etudes et de Recherche pour l'Intensification \\ du Traitement du Diabète (CERITD), Corbeil-Essonnes, France. \\ ${ }^{d}$ Diabeloop SA, 155 Cours Berriat, F-38000 Grenoble.
}

\begin{abstract}
This paper presents the first autoregressive with exogenous input (ARX) model using energy expenditure, carbohydrates on board, and insulin on board as input to predict interstitial glucose (IG). The proposed model may be used for predicting IG even during physical activity (PA). A populationbased model, obtained from a first database composed of 14 type 1 diabetes (T1D) patients, achieved a root-mean-square error (RMSE) of $16.7 \pm 15.6$ $\mathrm{mg} / \mathrm{dL}$, on IG prediction (30-min ahead) at the end of a PA, on a second database (15 T1D patients). Patient-specific ARX models, obtained on the second database, improved prediction accuracy $(\mathrm{RMSE}=7.8 \pm 4.5 \mathrm{mg} / \mathrm{dL})$, outperforming the results found in the literature.

Keywords: Interstitial glucose prediction, Physical activity, Energy expenditure, Meal, Insulin, T1D.
\end{abstract}

\section{Introduction}

Type 1 Diabetes (T1D) is a disease, where the insulin-producing cells (beta cells) are destroyed by the autoimmune system, causing a failure on blood glucose (BG) control. BG $>300 \mathrm{mg} / \mathrm{dL}$ may lead to fatigue, nausea, abdominal pain, excessive thirst, frequent urination and blurred vision.

C 2019 published by Elsevier. This manuscript is made available under the CC BY NC user license https://creativecommons.org/licenses/by-nc/4.0/ 
Chronic hyperglycemia (BG $>180 \mathrm{mg} / \mathrm{dL}$ ) may lead to long-term complications affecting eyes, kidneys, nerves and particularly the cardiovascular system. BG $<70 \mathrm{mg} / \mathrm{dL}$ (hypoglycemia) may lead to seizures, coma, and death. In fact, glucose is absorbed into the bloodstream after digestion of carbohydrates (CHO) in a meal, i.e., meals provoke an increase in BG. Glucose is also produced by the liver. Insulin is a hormone that allows glucose in the bloodstream to enter into cells, providing them with the energy they need to function, i.e., insulin provokes a decrease in BG. In this sense, the challenge for patients with T1D is to correctly dose their insulin administration in order to maintain their BG level into a target range, typically BG $\in[70,180] \mathrm{mg} / \mathrm{dL}$.

Interstitial glucose (IG) prediction plays an important role for automatically maintaining BG level of T1D patients into the targeted range. For instance, suspending insulin delivery when predicted IG is lower than a given threshold, allows reduction in hypoglycemic events $[1,2]$. As an other example, Model Predictive Control (MPC) algorithms use predicted IG to optimize insulin delivery $[3,4]$. In fact, a recently CE marked artificial pancreas (CE marking is a symbol of free marketability in the European Economic Area), uses IG predictions for regulating BG. For more details on this promising technology see [5] and [6]. The reader shall notice, that in this paper we make the difference between IG and BG. IG, which is provided by a continuous glucose monitoring (CGM) system and is highly correlated to BG [7], is the measure used by the artificial pancreas to regulate BG.

There exists a wide variety of models for predicting IG from a variety of input variables. In [8], three different model types: autoregressive (AR) models, AR models with exogenous input (ARX) and models based on an artificial neural network (ANN), were proposed. The AR-based models proposed in [8] only use IG information to perform IG prediction, whereas ARX and ANN-based models proposed in [8] use IG and insulin information. In [9], the predictive models receive as input variables IG, meal and insulin data. The models consist of a state-space model, an ARX model and an ARMAX model (autoregressive moving average with exogenous input). In $[10,11]$, two continuous-time second-order transfer functions are used with one using IG and injected insulin as inputs and the other using IG and amount of CHO of a meal. In [12], a hybrid model combining physiological (insulin and meal sub-models) and black box models (glucose-insulin interaction model and interstitial-continuous glucose monitoring model), was proposed. In [13] and [14] autoregressive integrated moving-average (ARIMAX) models are used 
into an MPC algorithm. The two ARIMAX models use IG and injected insulin as inputs to predict IG. Prediction horizon in [13] was $100 \mathrm{~min}$. In [14] prediction horizon was set to 10 hours. In [15], a state-space model, receiving insulin and IG as inputs, is used to predict IG in a horizon of 45 min. Prediction is used by an MPC algorithm to optimize insulin delivery.

Previous works are very interesting, but in their IG prediction models, no physical activity (PA) information (level, type, or sensor data) was considered as input variable. However, it is well-known that PA has a considerable effect on BG [16]. Authors in [17] demonstrated that the effect of PA on BG depends on the type (aerobic or resistance) and the intensity of the exercise. While aerobic physical activities induce a decrease on BG, resistance exercises induce an increase. Authors in [18] use a PA tracking watch to identify the "net" effect of idle, mild, moderate and intensive PA on BG. The aim of these studies was not to physiologically model the effect of PA into BG due to the complexity of this task. In fact, PA provokes an increase of blood flow in heart, lungs and peripheral tissue and a decrease of flow of kidneys and splanchnic organs [19, 20]. Peripheral glucose, insulin uptake, and liver's glucose production are increased during PA [20]. Glycogen depletion and replenishment are also affected by the intensity of PA [20]. Moreover, it is well known that insulin sensitivity is also affected during and after PA [21,22]. Two physiological models considering most of these effects were proposed in [20] and [23]. In [20] PA level is measured as a volume percentage of the maximum oxygen consumption $\left(\mathrm{VO}_{2}^{\mathrm{MAX}}\right)$. Depending on the $\mathrm{VO}_{2}^{\mathrm{MAX}}$, redistribution of blood flow, periphery glucose uptake, hepatic glucose production, and periphery insulin uptake are modulated in their model. In [23], PA level is measured indirectly using the heart rate (HR). Depending on the HR, insulin-independent glucose clearance, insulin sensitivity (up to 22 hours), and glucose uptake are modulated in their model. Although these physiological models are very interesting, validation on real patients was never performed. The reader shall notice that, it is very difficult to quantify the effect of PA on the physiological variables affecting the BG behaviour. In fact, this effect depends on a large variety of factors such as body weight, age, sex, physiological condition, patient training level, PA type and intensity [16], [21].

System identification is an alternative solution already used for considering the effect of PA in IG prediction. For instance, in [24], a subspace-based patient-specific model is proposed for IG prediction on T1D patients during 30 min of exercise. The model receives $\mathrm{CHO}$, insulin, HR, and respiration 
rate as inputs. In their model, PA is estimated by using $\mathrm{HR}$, however, it is well known that HR is also modulated by stress [25]. This fact may affect IG prediction accuracy in some situations. In [26], Dasanayake et al. proposed, a state-space model, which only receives IG and accelerometer signals as inputs. However, their model is only accurate, on IG prediction during PA, when heart rate is higher than $30 \%$ of the heart rate reserve $\left(\mathrm{HR}^{\mathrm{r}}\right)$. In [27], an hybrid model uses as inputs the meal and insulin information, and rate of perceived exertion (to consider PA). Since PA is considered through the patient perception, model performance may be affected. In [28], a model using insulin on board, energy expenditure (computed from accelerometer and HR signals) and galvanic skin response as inputs was proposed. The model, consisting of an ARMAX model, does not receive meal information as input. Therefore, IG prediction accuracy after meals may decrease.

In this paper, we propose an ARX model that uses energy expenditure (EE), insulin on board (IOB), and carbohydrates on board (COB), as inputs for predicting IG. EE, computed from both accelerometers and HR signals [29], is used to better consider the effect of PA on IG prediction, as demonstrated in [28] and [30]. IOB is computed from the output of an insulin pump. COB is computed from the CHO declared by the patients. Differently to the ARMAX model used in [28], the ARX model proposed in our paper includes the COB as input. This fact, allows to consider the effect of CHO, usually ingested before and during PA to prevent hypoglycemia, in order to improve prediction. Another difference between the ARMAX model presented in [28] and our ARX model is the intended use. While the ARMAX model proposed in [28] was designed to be used in an artificial pancreas that does not require meal announcement, the ARX model proposed in our paper is designed to be used in a hybrid closed-loop artificial pancreas. We could discuss the advantages and disadvantages of both approaches (meal announcement vs unannounced meal), but this is not the aim of this paper. The aim of this paper is to improve IG prediction during and after physical activities.

In this sense, originality of the proposed ARX model is the fact of using EE, IOB, and COB as inputs variables. We consider that the use of these three variables, usually modulated during (EE, IOB, COB) and after (IOB, COB) a PA, may improve IG predictions.

The rest of the paper is organized as follows: Section 2 presents a detailed description of the experimental protocols to acquire the two databases used in this paper. Section 3 describes the proposed ARX model, and the validation tests. Results, presented in Section 4, are discussed in Section 5. Finally, 
Section 6 presents the conclusions of the study.

\section{Database description}

Two different databases were used in this paper to estimate and validate the proposed ARX model. The first database was acquired from a clinical protocol where patients performed a single PA, namely "SPA protocol". The second database was acquired from a clinical protocol where patients performed four PAs, namely, "FPA protocol". These protocols were approved by the "French Ethics Committee" and the "French National Agency for Medicines and Health Products Safety (ANSM)".

\subsection{SPA database description}

T1D patients $(\mathrm{N}=35$, age $>18$ years old, $\mathrm{HbA} 1 \mathrm{c}<10 \%)$ already treated by insulin pump, were included in the clinical protocol, which was performed on 7 centers in France, in 2012.

After two visits, inclusion analysis and installation/calibration of two continuous glucose monitoring systems (Dexcom ${ }^{\circledR}$ SEVEN ${ }^{\circledR}$ PLUS), patients were hospitalized during 25 hours. Fig. 1 illustrates the SPA experimental procedure.

Patients arrived at 18:30 in the afternoon. An intravenous catheter, an insulin pump (JewelPUMP ${ }^{\mathrm{TM}}$ ), an accelerometer (hip-worn GT3X+, ActiGraph), and a PA monitoring system (Actiheart, CamNtech) were placed. Meals were taken at fixed hours (20:00, 8:00 and 12:00). Patients performed a PA at 15:00 during 30 min. The required PA, which consists of a step test, was performed at moderate intensity according to each patient. From the 35 initial patients, fourteen patients wearing the accelerometer and the HR monitoring system were included on this study. According to the proposed PA protocol, patients reduced basal insulin rate during the half hour of PA +2 hours. Moreover, when patient risked hypoglycemia (based on current and previous CGM measures), snacks were ingested and declared by patient.

Fig. 2 shows, for one patient of the SPA database, data set acquired during the second day of visit 3 of this experimentation. We can observe that 1) HR and counts per minutes (CPM), increase during PA (PA started at 15:00), 2) for this patient, HR and CPM also increase between 10:00 and 11:00, which indicates that this patient performed an undeclared PA, 3) meal have an important and delayed effect on IG (IG increases around $40 \mathrm{~min}$ after meal), 4) as already mentioned, insulin basal rate is reduced 


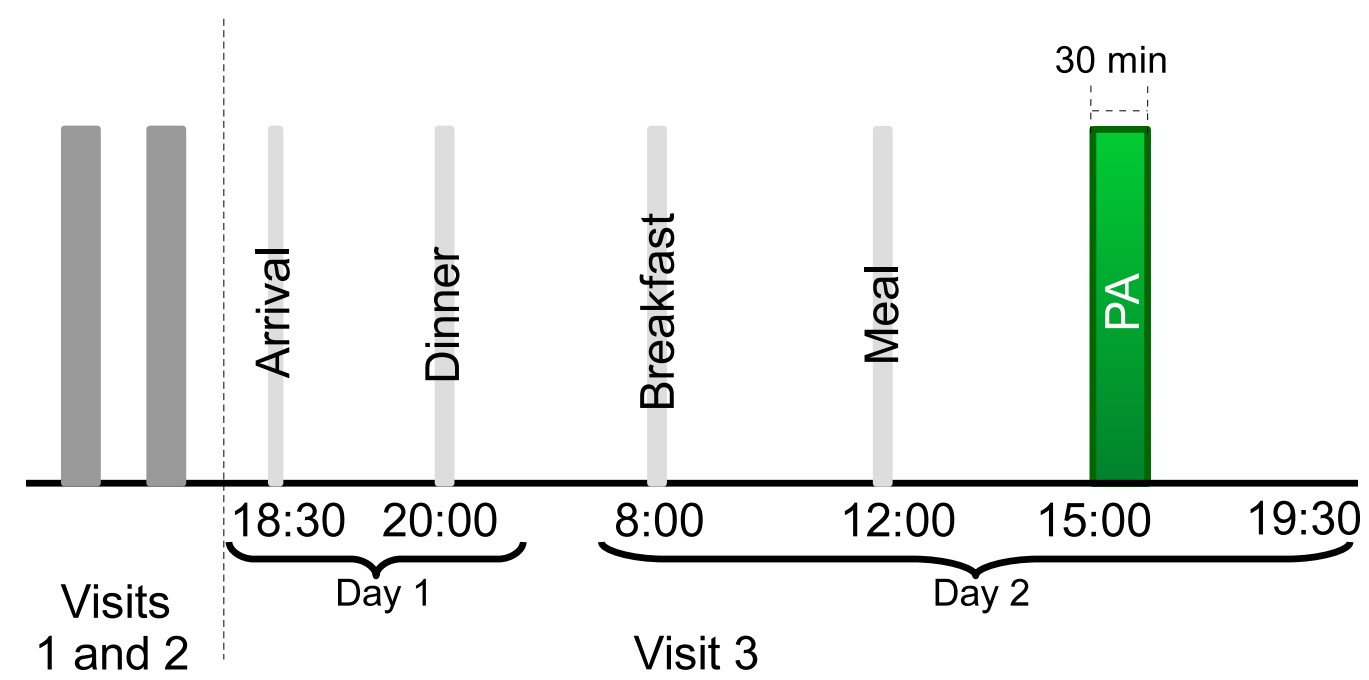

Figure 1: SPA study procedure was performed on visit 3. Subjects arrived at 18:30 and took dinner at 20:00 of day 1 of visit 3. On day 2 of visit 3, patients took breakfast and meal at 8:00 and 12:00, respectively, and performed a PA at 15:00 during 30 min.

when the patient started the PA in order to prevent hypoglycemia. More precisely, the aim of Fig. 2 is to illustrate data set used in this work to estimate (order selection and parameter estimation) the ARX model, i.e., data set $\in\left[t_{\mathrm{PA}}-360, t_{\mathrm{PA}}+120\right]$ min, where $t_{\mathrm{PA}}$ is the time at which PA was started. This time interval was used on all the patients of the SPA database.

\subsection{FPA database description}

T1D patients $(\mathrm{N}=36$, age $>18$ years old, $7.5 \%<\mathrm{HbA1c}<9.5 \%)$, already treated by insulin pump, and able to practice at least one PA during 3 days, were included in the clinical protocol, which was performed in 9 centers in France, in 2016.

This study was performed in 3 visits (see Fig. 3). During the first visit, inclusion was performed, CGM system (Dexcom ${ }^{\mathrm{TM}}$ Share AP, Dexcom Inc., San Diego, CA) was installed and calibrated, patients were instructed on the CGM system utilization, patients were randomized on 2 groups, and dates for visits 2 and 3 were established.

Visit 2 was done two days before the main visit (V3). An accelerometer (hip-worn GT3X+, ActiGraph), and a PA monitoring system (Actiheart, CamNtech) were placed. 


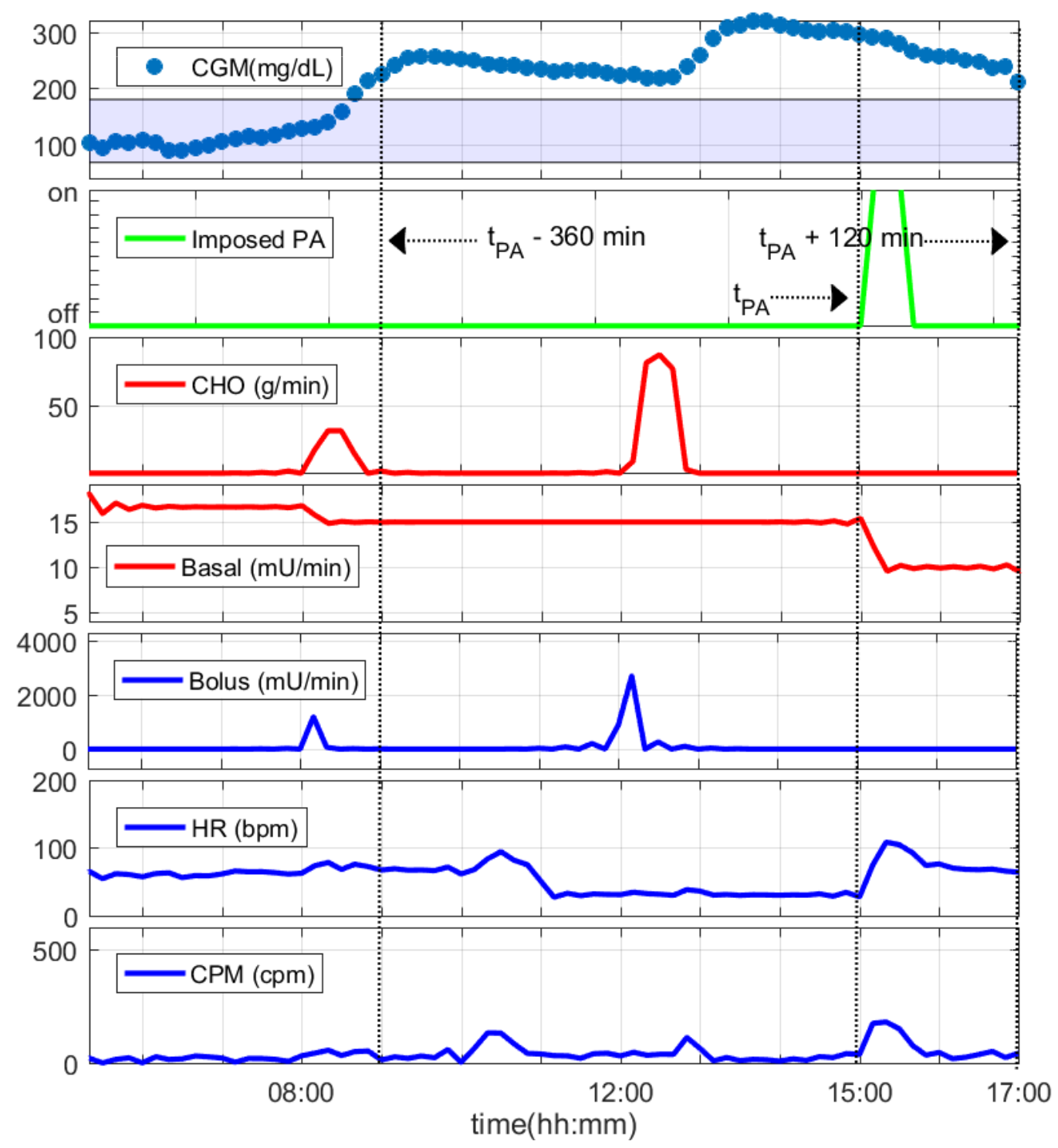

Figure 2: Data set (of a SPA database patient) used in this work for modeling (from 5:00 in the morning to 17:00 in the afternoon). Breakfast, and meal were taken at 8:00, and 12:00, respectively. Patients performed a PA at 15:00 during 30 min. 


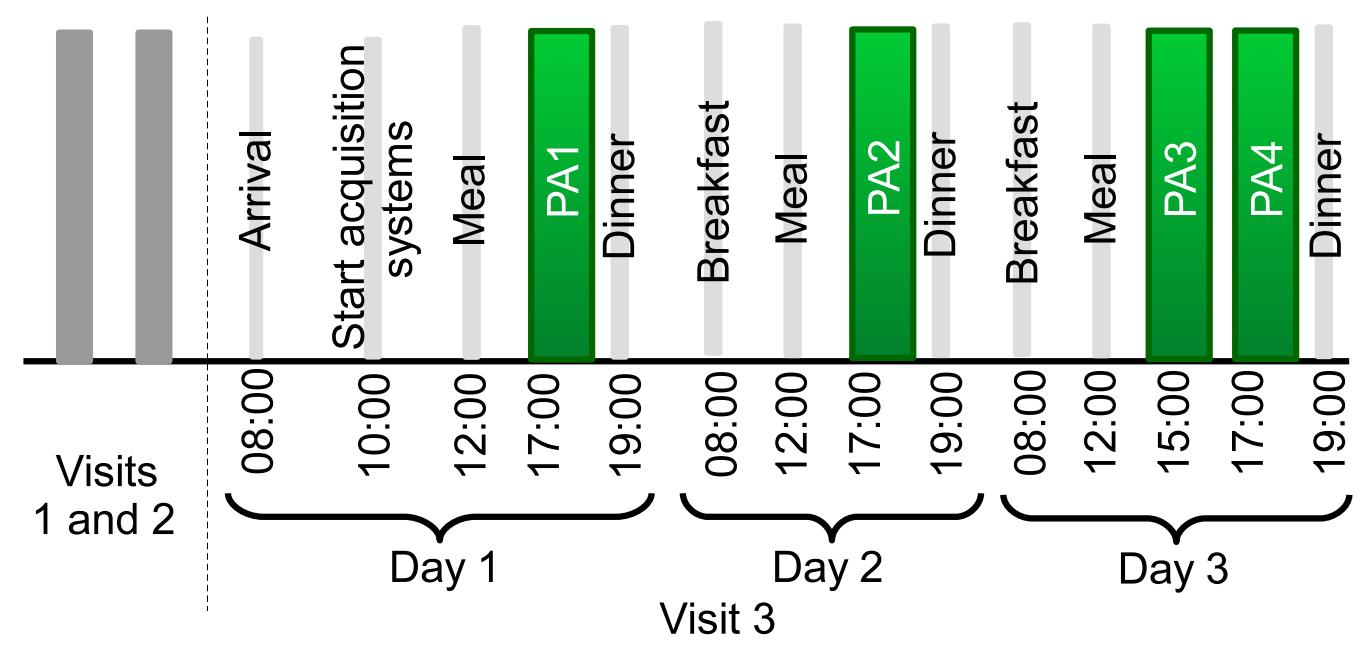

Figure 3: FPA study procedure was performed on 3 visits. During the main visit patients arrived at 8:00 and stayed in the research center during 3 days, and performed 4 physical activities (PA1, PA2, PA3, and PA4).

Concerning the visit 3 (see Fig. 3), patients arrived to the research center at 8:00 (after taking breakfast at home), and spent 72 hours in the research center. If patients were in group A, they used a closed-loop Diabeloop artificial pancreas, i.e., the CGM Dexcom ${ }^{\mathrm{TM}}$ Share AP, the smartphone Samsung (integrating the Diabeloop AP algorithm), and the insulin pump Cellnovo. If patients were in group B, they used open loop, i.e., the CGM Dexcom ${ }^{\mathrm{TM}}$ Share AP and, their own insulin pump and usual treatment. Acquisition systems were started at 10:00.

During visit 3, patients performed daily physical activities, but also some imposed physical activities. Meals, of various $\mathrm{CHO}$ quantities, were taken at the same hours during the three days. Imposed physical activities were performed at fixed hours. Intensity and duration of physical activities were not the same during the three days of the visit. When PA started $3 \mathrm{~h}$ after meal, each patient reduced the insulin basal rate at 50 or $80 \%$ of the current basal rate, depending on the PA intensity, 30 to 60 min before starting the PA. When PA started within the $3 \mathrm{~h}$ after meal, bolus correcting meal was reduced. When required (hypoglycemia risk), snacks were ingested and declared by the patient. From the 36 initial patients, fifteen patients wearing the accelerometer and the heart rate monitoring system were included on this study. 
Fig. 4 displays, for one patient of the FPA database, data set acquired during the 3 days of visit 3 . We can observe that patients performed a PA during day 1 (PA1), a PA during day 2 (PA2), and two PAs during day 3 (PA3 and PA4). We can also observe that when patient risked hypoglycemia, snacks were ingested (see small increases in $\mathrm{CHO}$, in the third $\mathrm{CHO}$ panel).

In both protocols (SPA and FPA), CGM calibration was performed 1) at the installation phase by two BG measurements, 2) when instructed by the 12-hour CGM calibration prompt, and 3) when the CGM reading was inaccurate. In SPA protocol and the main visit of FPA protocol, BG was measured, by a glucose meter, at least every hour, but also every $15 \mathrm{~min}$ during meals, PA, hypoglycemia, and hyperglycemia.

In both protocols (SPA and FPA), IG was acquired every 5 min, declared PA, declared CHO, insulin basal rate, bolus, and HR (computed from electrocardiogram) were sampled every min. Accelerometer signals were converted in counts per minute. Finally all the signals were preprocessed and re-sampled to a sampling period of $10 \mathrm{~min}$, which is the sampling period used in the proposed ARX model.

The 14 patients of the SPA protocol and the 15 patients of the FPA protocol, used in this study (good quality of CGM, CPM, and HR signals), were different. In fact, SPA and FPA protocols were performed on different years (2012 and 2016).

In both studies (SPA and FPA), CHO and PA type, were declared by the patients.

\section{The proposed ARX model}

This section presents the ARX model proposed in this paper. Differently to the black box models found in the literature (for instances [28, 30, 26, 24]), the proposed ARX model uses EE, IOB, and COB as inputs to improve IG prediction. These inputs allow to consider 1) the intensity and duration of a PA, 2) the delivered insulin which is modulated (before and during PA) to reduce the risk of hypoglycemia, and 3) the $\mathrm{CHO}$, usually ingested before and during PA to prevent hypoglycemia. Notice that these are important factors affecting BG dynamic. 


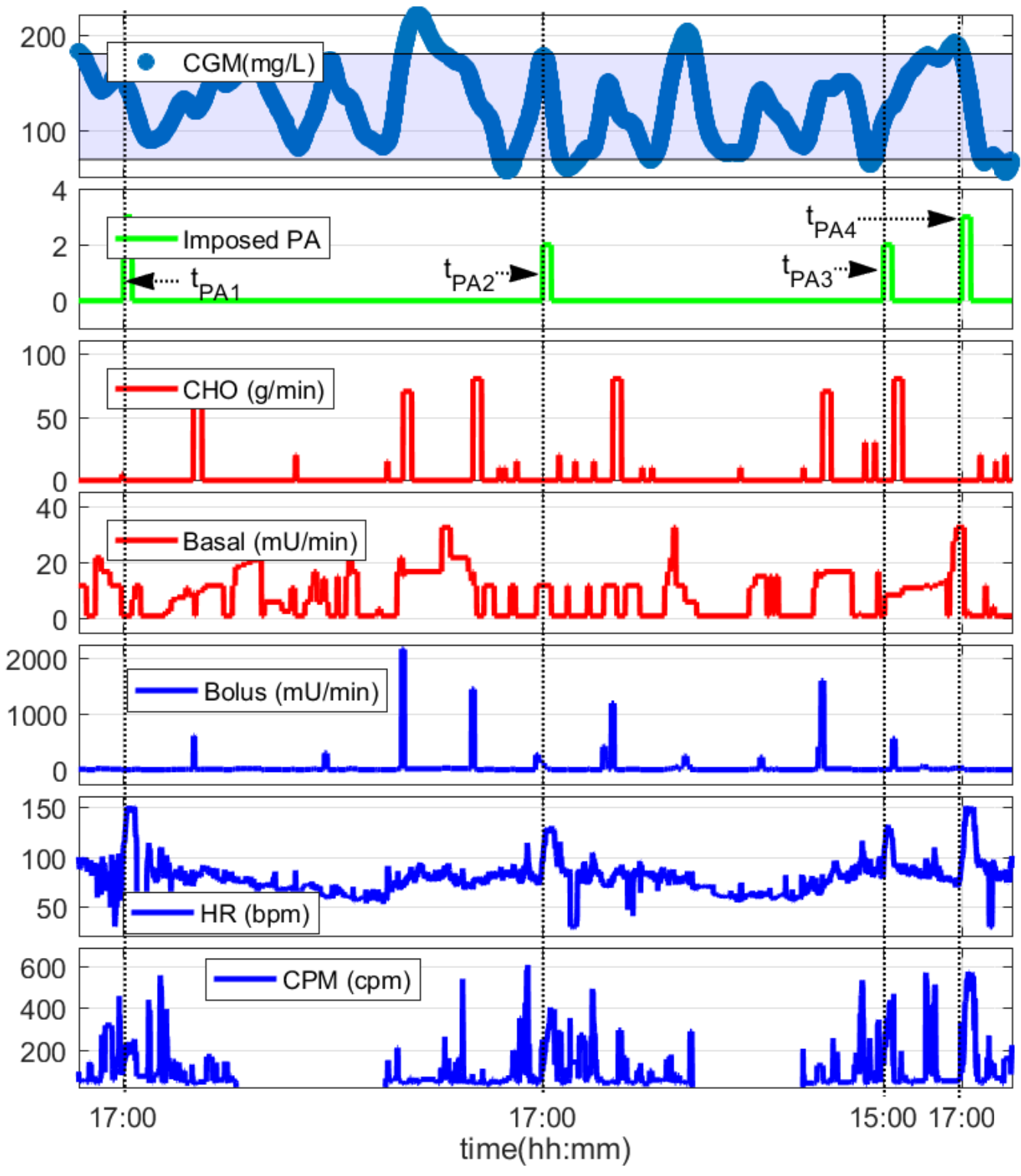

Figure 4: Data set (of a FPA database patient) used in this work for modeling (estimation and/or validation). Initialization was performed at 10:00. Breakfast, meal and dinner were taken at 8:00, 12:00, and 19:00, respectively. Patients performed a PA at 17:00 during 30 min or 45 min. Patients also performed a PA at 12:00 of day 3. 


\subsection{ARX structure}

In system identification, ARX models are among the most used black box structures due to their simplicity $[31,32]$. The ARX model is given by (1).

$$
y[k]=\mathbf{a}^{T} \mathbf{y}_{k-1}+\mathbf{b}^{T} \mathbf{u}_{k-n k}+e[k]
$$

where $k$ is the current sample. Both $\mathbf{a}=\left[a_{1}, \ldots, a_{n a}\right]$ and $\mathbf{b}=\left[b_{1}, \ldots, b_{n b}\right]$ are the model parameters. The regressors $\mathbf{y}_{k-1}=[y[k-1], \ldots, y[k-n a]]$ are the previous outputs on which the current output $(y[k])$ depends. The regressors $\mathbf{u}_{k-n k}=[u[k-n k], \ldots, u[k-n k-n b+1]]$ are the delayed inputs on which the current output depends. The parameters $n a$ and $n b$ are the orders of the ARX model, $n k$ is the time delay (expressed in samples) before the input affects the output, also called the dead time of the system. Finally, $e[k]$ is a noisy value.

\subsection{Proposed ARX model structure}

ARX models may be used for representing multiple-input and singleoutput (MISO) systems. In this paper, the proposed ARX model, given by (2), receives as inputs the IOB computed according to (3), the COB computed according to (4), and the EE computed according to (6). The black box model in (2) will determine the temporal relations that may exist between the inputs IOB, COB, EE and the output IG. Model parameters are estimated from MISO data in order to dynamically represent the effect of inputs into IG.

$$
\hat{y}^{\mathrm{IG}}[k]=\mathbf{a}^{T} \mathbf{y}_{k-1}^{\mathrm{IG}}+\mathbf{b}^{T} \mathbf{u}_{k-n k_{1}}^{\mathrm{EE}}+\mathbf{c}^{T} \mathbf{u}_{k-n k_{2}}^{\mathrm{IOB}}+\mathbf{d}^{T} \mathbf{u}_{k-n k_{3}}^{\mathrm{COB}}
$$

The ARX model, proposed in this paper, used a sampling period of $10 \mathrm{~min}$, according to previous works found in the literature $[9,28]$. However, predictions may be performed every 5 min (sampling period of $\mathrm{IG}$ ), i.e., every 5 min the ARX model resamples the past signals to $10 \mathrm{~min}$ in order to predict IG $[10,20,30, \cdots, N]$ min ahead. In this paper we present examples of 30 , 60 and $120 \mathrm{~min}$ ahead IG predictions.

\subsection{Model inputs}

3.3.1. IOB input

The IOB refers to the injected insulin (bolus and basal), that is still to have an effect on the BG. The IOB is computed as a convolution:

$$
u^{\mathrm{IOB}}[n]=\sum_{k=0}^{K} I[n-k] h_{\mathrm{IOB}}[k]
$$


where $I[k]$ is the quantity of insulin in $\mathrm{mU}$ delivered by the insulin pump at the $k$-th time index.

\subsection{2. $C O B$ input}

In the same spirit, the $\mathrm{COB}$ refers to the portion of the meal that is still to have an effect on the IG. The COB is computed as a similar convolution:

$$
u^{\mathrm{COB}}[n]=\sum_{k=0}^{K} C H O[n-k] h_{\mathrm{COB}}[k]
$$

where $C H O[k]$ is the quantity of $\mathrm{CHO}$ in $\mathrm{g}$ ingested at the $k$-th time index. As already mentioned, $\mathrm{CHO}$ were declared by the patients.

In (3) and (4), $K=144$ to consider $24 \mathrm{~h}$ of data, and $h[k]=h\left[k \tau_{s}\right]$ with $\tau_{s}=10 \mathrm{~min}$, is given by (5):

$$
h[k]=\left[1+\frac{k}{\tau}\right] e^{-\frac{k}{\tau}} .
$$

$\tau=50 \mathrm{~min}$ in (3) was set from a population-based study (SPA protocol), where insulinemia was measured every 10 min during a given period. $\tau=40$ min in (4) was empirically set. We considered the fact that CHO have usually a faster effect on IG than insulin.

\subsubsection{EE input}

The EE is computed from accelerometer and HR signals, according to (6).

$$
u^{\mathrm{EE}}= \begin{cases}\alpha_{1} \mathrm{HR}^{\mathrm{r}}+\beta_{1}, & \text { if } \mathrm{HR}^{\mathrm{r}} \geq S_{\mathrm{HR}^{\mathrm{r}}}, \\ \alpha_{2} \mathrm{LC}+\beta_{2}, & \text { if } \mathrm{HR}^{\mathrm{r}}<S_{\mathrm{HR}^{\mathrm{r}}} \text { and } \mathrm{LC}<S_{\mathrm{LC}}, \\ \alpha_{3} \mathrm{LC}+\beta_{3}, & \text { if } \mathrm{HR}^{\mathrm{r}}<S_{\mathrm{HR}^{\mathrm{r}}} \text { and } \mathrm{LC} \geq S_{\mathrm{LC}} .\end{cases}
$$

where $\alpha_{1}=5.45, \beta_{1}=-66.09, \alpha_{2}=256.09, \beta_{2}=-0.13, \alpha_{3}=85.99$, $\beta_{3}=82.39$ are the model parameters. $S_{\mathrm{HR}^{\mathrm{r}}}=40 \mathrm{bpm}$ and $S_{\mathrm{LC}}=0.5$ are the cut points obtained from a population-based approach. LC is a linear combination of the normalized values of $\mathrm{HR}^{\mathrm{r}}$ and $\mathrm{CPM}$, computed as:

$$
\mathrm{LC}=\theta_{1} \mathrm{CPM}+\theta_{2} \mathrm{HR}^{\mathrm{r}} .
$$

where $\mathrm{HR}^{\mathrm{r}}=\mathrm{HR}$ - resting $\mathrm{HR}, \mathrm{CPM}$ are the counts per minute (a quantity derived from the accelerometer signal [33]).

Notice that, in this model, PA information declared by the patients is not used to compute EE. For more details on model (6) see [29]. 


\subsection{Model orders and delays}

The orders ( $n a, n b, n c$, and $n d)$ and delays $\left(n k_{1}, n k_{2}\right.$, and $\left.n k_{3}\right)$ of the ARX model proposed in this paper, were obtained by a standard system identification methodology, described in the following.

1) $n a, n b, n c, n d, n k_{1}, n k_{2}$, and $n k_{3}$ are changed among a given range of values,

2 ) each time that $n a, n b, n c, n d, n k_{1}, n k_{2}$, or $n k_{3}$ changes, model parameters are estimated, by the classical least squares algorithm (7), and an associated Akaike final prediction error $(F P E)$ is computed by (8).

$$
\hat{\theta}=\left(\mathbf{X}^{T} \mathbf{X}\right)^{-1} \mathbf{X}^{T} \mathbf{y},
$$

where $\mathbf{X}$ is the regression matrix, $\mathbf{y}$ is a $N \times 1$ vector of outputs (IG in this case), and $\hat{\theta}$ represents the estimated parameters.

$$
F P E=\frac{1+d / N}{1-d / N}\left(\frac{1}{N} \mathbf{e}(k, \hat{\theta})(\mathbf{e}(k, \hat{\theta}))^{T}\right),
$$

where $N$ is the number of values in the estimation data set, $\mathbf{e}(t, \hat{\theta})$ is a vector of prediction errors, and $d$ is the number of estimated parameters $(\hat{\theta})$.

3) the model structure (orders and delays) leading the lowest final prediction error is chosen as the best candidate. Notice that by using this selection criteria (8) the overparametrization is penalized.

Database used for choosing the model structure (orders and delays) was the SPA database. Datasets into the span $\left[t_{\mathrm{PA}}-360 \mathrm{~min}, t_{\mathrm{PA}}+120 \mathrm{~min}\right]$ sampled at $\tau=10 \mathrm{~min}$, were used for training.

Accordingly with the system identification procedure described above: 1) model orders and delays were changed in the ranges: $n a \in[1,10], n b \in$ $[7,8], n c \in[10,15], n d \in[10,15], n k_{1} \in[1,2], n k_{2} \in[1,2], n k_{3} \in[1,3] ;$ 2) each time that a given set of orders and delays was set: a) a regression matrix was constructed for each patient, b) the regression matrix of aull patients was concatenated, c) a model (parameters) was estimated, by the least squares algorithm, and d) a final prediction error associated with such model is computed; 3) finally, the model structure (orders and delays $n a=3$, 
$n b=5, n c=11, n d=7$, and $\left.n k_{1}=n k_{2}=n k_{3}=2\right)$, yielding the lowest final prediction error was chosen as the best candidate. It is interesting to notice that the selected model structure allows to consider the effect of IOB in the interval [t - $130 \mathrm{~min}, \mathrm{t}-20 \mathrm{~min}]$, and the effect of COB in the interval $[\mathrm{t}$ $90 \mathrm{~min}, \mathrm{t}-20 \mathrm{~min}$. These time intervals cover the periods where insulin [34] and $\mathrm{CHO}[35]$ have the most important effect on BG.

\subsection{ARX validation tests}

The goals of the validation tests presented in this paper are 1) to evaluate the possibility of proposing a population-based ARX model, 2) to verify the hypothesis on ARX models stating that performance of personalized ARX models may increase as the number of quality-training-data increases, and 3 ) to test the improvement achieved by using the three regressors.

\subsubsection{Test 1: The population-based ARX model obtained from the SPA database is evaluated on the FPA database}

As a first approach, we evaluated the possibility of using an ARX model for predicting IG in any T1D adult patient. In this sense, ARX parameters $(\mathbf{a}, \mathbf{b}, \mathbf{c}$, and $\mathbf{d}$ in (2)) are obtained from data sets on which model orders and delays were chosen. Similar to the model structure selection, the concatenated regression matrix was used to obtain the SPA population-based ARX parameters. Then, the population-based (PB) model was used for predicting IG on the 15 patients of the FPA database, during 30, 60 and $120 \mathrm{~min}$, after the physical activity (PA3) was started (see Fig. 4).

\subsubsection{Test 2: Increasing ARX performance by increasing training data}

We hypothesize, according to literature [36], that black box models performance may increase, if the number of quality-available-training data increases. In this sense, patient-specific ARX models were obtained on the FPA database from:

a) a single data set (T1), i.e., data around PA1, PA2, or PA4 is used separately for training;

b) on two data sets (T2), i.e., data around PA1 and PA2, PA1 and PA4, or PA2 and PA4 is used for training;

c) on three data sets (T3), i.e., data around PA1, PA2, and PA4 is used for training. 
We refer to data around a given PA, as a set of 8 hours of data into the $\operatorname{span}\left[t_{\mathrm{PAi}}-60, t_{\mathrm{PAi}}+360 \mathrm{~min}\right]$, where $t_{\mathrm{PAi}}$ is the time at which one of the physical activities PA1, PA2 or PA4 was started. In this sense models T1 are trained on 8 hours of data, T2 models are trained on 16 hours of data, and T3 models are trained on 24 hours of data.

Finally, T1, T2, and T3 ARX models were used for predicting IG, into the span $\left[t_{\mathrm{PA} 3}, t_{\mathrm{PA} 3}+30 \mathrm{~min}\right],\left[t_{\mathrm{PA} 3}, t_{\mathrm{PA} 3}+60 \mathrm{~min}\right]$, and $\left[t_{\mathrm{PA} 3}, t_{\mathrm{PA} 3}+120 \mathrm{~min}\right]$.

\subsubsection{Test 3: Improvement achieved by the use of the three variables (insulin,} meal, and EE)

On the FPA database, we obtained 1) T3 models using COB, IOB, and EE as inputs, 2) T3 models using only COB and IOB as inputs (NEE), 3) T3 models using only EE and IOB as inputs (NCOB), and 4) T3 models using only EE and COB as inputs (NIOB). Models T3, NEE, NCOB, and NIOB, are compared in order to show the improvement reached by the use of three simultaneous variables as inputs of the ARX models.

\subsection{Performance indicator}

Performance indicator used for measuring model accuracy is the rootmean-square error (RMSE), given by:

$$
\mathrm{RMSE}=\sqrt{\frac{1}{N} \sum_{n=1}^{N}\left(y^{\mathrm{IG}}[n]-\widehat{y}^{\mathrm{IG}}[n]\right)^{2}},
$$

which is a standard indicator used in IG prediction $[8,9,10,11,12]$. In (9), $y^{\mathrm{IG}}[n]$ and $\widehat{y}^{\mathrm{IG}}[n]$, are the measured and predicted IG at instant $n$, respectively. Since in tests described above the ARX models are used for predicting IG during 30, 60, and $120 \mathrm{~min}$, and the sampling period was $10 \mathrm{~min}$, then $N=3,6$, and 12 , respectively.

P-value, computed by Wilcoxon Matched-Pairs signed-rank test [37], which is a non-parametric statistical hypothesis test, was used for validation on test 3, described above.

\section{Results}

Parameters of the proposed PB ARX model were $a 1=1.67, a 2=-0.74$, $a 3=0.06, b 1=-1.03, b 2=1.87, b 3=-0.71, b 4=1.35, b 5=-0.12$, $c 1=3.39 e^{-4}, c 2=-1.09 e^{-4}, c 3=-0.34 e^{-4}, c 4=-3.02 e^{-4}, c 5=3.82 e^{-4}$, 

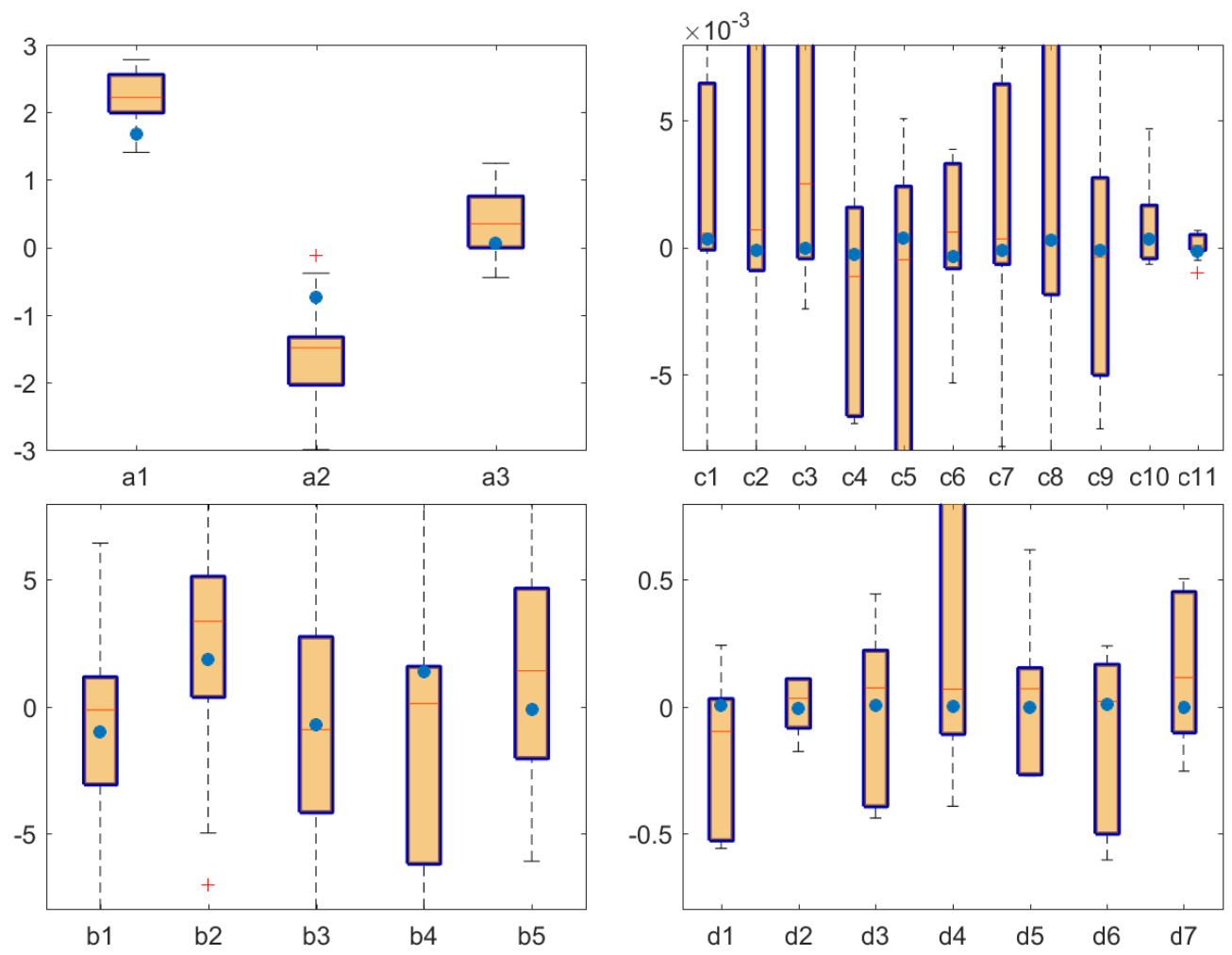

Figure 5: Parameters of the personalized ARX models derived from T3 models. Since parameters were personalized for 15 patients, each box is composed of 15 points, i.e., each parameter can take 15 values. Parameters of the PB model are also displayed (blue points), representing the value of each parameter.

$c 6=-3.73 e^{-4}, c 7=-1.19 e^{-4}, c 8=2.63 e^{-4}, c 9=-1.26 e^{-4}, c 10=3.37 e^{-4}$, $c 11=-1.74 e^{-4}, d 1=0.57 e^{-2}, d 2=-0.73 e^{-2}, d 3=0.45 e^{-2}, d 4=-0.08 e^{-2}$, $d 5=-0.59 e^{-2}, d 6=0.98 e^{-2}$, and $d 7=-0.54 e^{-2}$.

Fig. 5 displays the parameters of the 15 patient-specific ARX models (boxplots) obtained for T3 models. On the same figure, parameters of the population-based ARX model (PB-model) are also represented (single blue points).

We observe in Fig. 5 that all the parameters of the PB model are inside the boxplots, i.e., min and max values of each parameter of the T3 models. Moreover, the parameters $a_{3}, \mathbf{b}, \mathbf{c}$, and $\mathbf{d}$ are inside the interquartile range, i.e., first and third quartiles. We see that the coefficients $a_{1}, a_{2}$ are roughly opposite, which means that, as expected, the prediction is sensitive to the 
Table 1: RMSE (mean \pm standard deviation) computed for IG prediction during 30, 60 and 120 min, performed by models T3, T2, T1, and PB.

\begin{tabular}{cccc}
\hline Model & $\begin{array}{c}30 \mathrm{~min} \\
(\mathrm{mg} / \mathrm{dL})\end{array}$ & $\begin{array}{c}60 \mathrm{~min} \\
(\mathrm{mg} / \mathrm{dL})\end{array}$ & $\begin{array}{c}120 \mathrm{~min} \\
(\mathrm{mg} / \mathrm{dL})\end{array}$ \\
\hline T3 & $7.75 \pm 4.51$ & $15.86 \pm 9.61$ & $35.24 \pm 19.52$ \\
\hline T2 & $8.94 \pm 6.17$ & $19.11 \pm 12.24$ & $41.22 \pm 28.01$ \\
\hline T1 & $21.82 \pm 17.02$ & $81.78 \pm 120.61$ & $327.04 \pm 473.29$ \\
\hline PB & $16.70 \pm 15.56$ & $31.67 \pm 25.84$ & $44.50 \pm 30.45$ \\
\hline
\end{tabular}

Table 2: P-values, by Wilcoxon Matched-Pairs signed-rank test, from comparisons between RMSE reached by the model T3 and the models NEE, NCOB, and NIOB, on IG prediction during 30, 60 and 120 min.

\begin{tabular}{cccc}
\hline Model & $30 \mathrm{~min}$ & $60 \mathrm{~min}$ & $120 \mathrm{~min}$ \\
\hline NEE & 0.03 & 0.04 & 0.28 \\
\hline NCOB & 0.35 & 0.30 & 0.04 \\
\hline NIOB & 0.52 & 0.21 & 0.08 \\
\hline
\end{tabular}

current IG slope. Since the proposed ARX model is a black box model, more explanation on the meaning of the rest of parameters may not be given.

Fig. 6 (left) shows results of tests 1 and 2. Performance reached by the ARX models obtained from different training data sets are represented by boxplots T3, T2, and T1, respectively. Performance of the SPA populationbased ARX model is represented by boxplot PB. Table 1 presents (mean \pm standard deviation) RMSE on IG prediction during 30, 60 and $120 \mathrm{~min}$, performed by each model.

Fig. 6 (right) displays results of test 3, i.e., RMSE reached by models T3, NEE, NCOB, and NIOB. Table 2 displays p-values (obtained by Wilcoxon Matched-Pairs signed-rank test) of the comparison between the T3 models and the NEE, NCOB, and NIOB models, on IG prediction during 30, 60, and $120 \mathrm{~min}$.

Finally, Fig. 7 illustrates a representative example of $30 \mathrm{~min}$ ahead IG prediction, performed by the proposed T3 model (considering EE, IOB, and COB as inputs), on one patient of the FPA database. As already mentioned, even if the ARX model uses a sampling period of 10 min, IG prediction may be performed every $5 \mathrm{~min}$, as is the case in this example. 

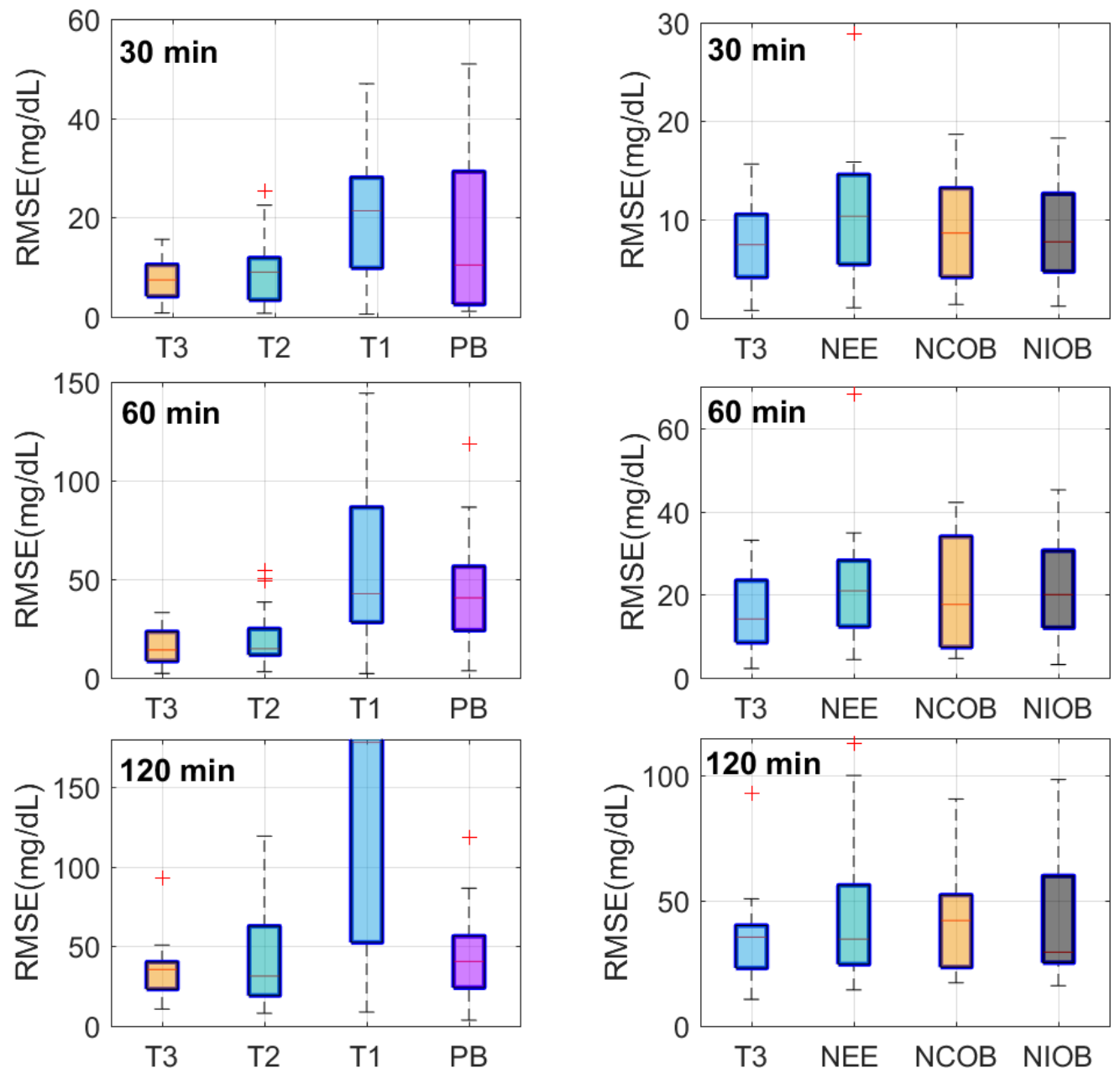

Figure 6: (left) Performance on IG prediction by ARX models trained on 1, 2, and 3 data sets, i.e., T1, T2, and T3, respectively. Model trained on the SPA population is also included (PB). Models are used for predicting IG during $30 \mathrm{~min}$ (top panel), 60 min (middle panel), and $120 \mathrm{~min}$ (bottom panel). (right) Performance on IG prediction by ARX models, trained on 3 data sets. Models T3 uses EE, COB and IOB as inputs. Models NEE uses COB and IOB. Models NCOB uses EE and IOB as inputs. Models NIOB uses EE and COB as inputs. The four models are used for predicting IG during 30 min (top panel), 60 min (middle panel), and 120 min (bottom panel). 


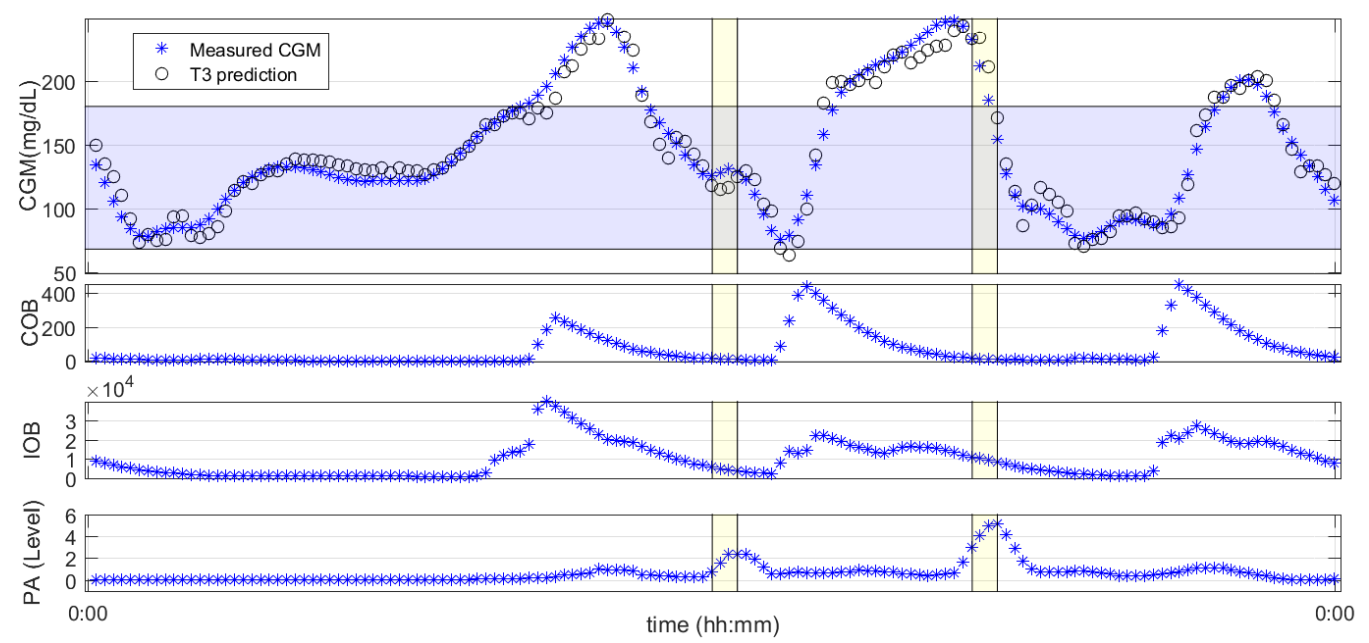

Figure 7: IG prediction 30 min ahead, by a patient-specific ARX model using as inputs EE, COB, and IOB (i.e. a T3 model). Every 5 min, the ARX model uses re-sampled signals (10 min) to predict IG $30 \mathrm{~min}$ ahead.

\section{Discussion}

Fig. 5 shows that parameters of the population-based ARX model, obtained from the SPA database, are within the ranges of parameters of the patient-specific ARX models obtained from the FPA database. Therefore, performance reached by the PB model is good (compared with the obtained patient-specific models) for IG predictions (during 30, 60, and $120 \mathrm{~min}$ ) on the FPA database. In fact, in [26], authors reported an average mean absolute error (MAE), over 15 patients, during 30 min of IG predictions (during PA) of $19.7 \mathrm{mg} / \mathrm{dL}$. The PB model proposed in this paper reached an average MAE of $14.93 \mathrm{mg} / \mathrm{dL}$, over 15 patients, during $30 \mathrm{~min}$ of IG prediction. Considering these results we can conclude that the proposed PB model is more accurate than the model presented in [26]) on 30-min IG predictions. But the two models were not developed under the same conditions and for the same goal: 1) the model that we propose in this paper allows IG prediction during and out of PA periods, whereas the model proposed in [26] is only accurate on PA periods; 2) the model proposed in this paper uses EE (from HR and accelerometer signals), IOB, and COB (from CHO declared by the patient) as exogenous inputs, whereas the model proposed in [26] only uses accelerometer signals; 3) experiments in [26] were different of those performed in this paper. In fact, this is the first time that SPA and FPA 
protocols are reported in a paper. For these reasons, a true comparison may not be established.

Fig. 6 (left) allows us to demonstrate that performance of patient-specific ARX models increases if the number of training data increases. We can observe that, for T3 models trained on 24 hours of data, the RMSE on IG prediction during 30, 60 and $120 \mathrm{~min}$ is lower than RMSE obtained by models T2 and T1, i.e., models trained on 16 and 8 hours of data, respectively. Table 1 confirms these results quantitatively. Mean RMSE reached by T3 models is three times lower than the one obtained by models T1 on IG prediction during $30 \mathrm{~min}$, five times lower on IG prediction $60 \mathrm{~min}$ ahead and nine times lower on IG predictions 120 min ahead. Comparing T3 and T2 models, difference in performance is less important than that observed while comparing T3 and T1 models, but T3 models remain more accurate.

Fig. 6 (right) shows that models using EE, COB and IOB as inputs are more accurate than those models using only IOB and COB, on 30 and 60 min ahead IG predictions. A p-value lower than 0.05 was found, when evaluating the difference between T3 and NEE models. However, a p-value $=0.28$ was found on IG prediction during $120 \mathrm{~min}$. These results may be interpreted as follows. When a T1D patient performs a PA, the use of EE as input in the models is very important (during PA and even 30 min after PA). However, when the effect of the performed PA is reduced (120 min after PA), the use of EE as input is less important, but, as showed in Fig. 6 (right), still allows improvement in performance. Concerning the effect of suppressing the COB input or the IOB input of the models, T3 models reached better performance than NCOB and NIOB models on the three prediction horizons, but difference was not significative.

Finally, Fig. 7 shows an example on 30 min ahead IG prediction, by the T3 model during a day (day 3 of visit 3 of the FPA protocol shown in Fig. 3 ). We can observe that the proposed model is good for predicting IG during PA but also out of PA. Therefore we consider that this model may be used on any application where $30 \mathrm{~min}$ ahead IG prediction is required, regardless of whether or not, the patients is performing a PA.

In fact, other works have reported results on 30 min ahead IG prediction by linear black box models. In [8] an AR model, using only IG information as input, reached a RMSE ranged between 14.0 and $21.6 \mathrm{mg} / \mathrm{dL}$, whereas an ARX model, using IG and insulin information as inputs, reached a RMSE ranged between 13.3 and $18.8 \mathrm{mg} / \mathrm{dL}$. These results are promising, however, estimation and validation were done on a virtual population, and the model 
was not confronted to the complex dynamics of IG on real T1D patients. In [9], a state-space model, receiving meal and insulin data as input variables, reached a $\mathrm{RMSE}=18.08 \mathrm{mg} / \mathrm{dL}$ on $\mathrm{IG}$ prediction $30 \mathrm{~min}$ ahead, on a real T1D patient. In [12] a model combining physiological and black box models, reached RMSE of 19.1, 19.5, and $21.1 \mathrm{mg} / \mathrm{dL}$ on IG prediction during 20, 40, and $60 \mathrm{~min}$, respectively. The reader shall notice that similar to our model, in these works insulin and IG information is automatically acquired by an insulin pump and a CGM system, respectively. CHO information is manually reported by the patients. However, experiments performed in these works (PA is not considered) are different to those performed in our study. Therefore, a comparison between results reported by the other works and ours may not be fair. But, RMSE reached by our models, using EE, COB, and IOB as inputs, trained on 24 hours of data (i.e., T3 models), on IG prediction during 30 and $60 \mathrm{~min}(\mathrm{RMSE}=7.75 \pm 4.51$ and $\mathrm{RMSE}=15.86$ \pm 9.61 , respectively), on $15 \mathrm{~T} 1 \mathrm{D}$ patients, show the interest of this paper.

\subsection{Limitations}

The main limitation of the study is that models were performed and validated on two databases composed of adult patients. Then, we can not assure that proposed models will accurately predict IG on children or adolescent patients. Other limitation of the proposed model is the inability to accommodate for disturbances that may occurs in the prediction horizon. In fact, predictions are based on past and current data, then future meals and physical activities will not be considered on the IG prediction. Other limitation is the fact of using IOB and COB time constants at fixed values (one population-based and the other empirically chosen). In fact, these time constants, which are patient dependent variables, may affect IG prediction performed by the proposed models. Another, limitation of the proposed model is that insulin sensitivity, which is also a patient dependent variable that varies during the day, is not considered by the model. This limitation may be overcome by adapting the model parameters during the day [8]. Finally, a limitation concerns the heart rate and accelerometer sensors errors. For instance, in Fig. 4 (bottom panel) some sensors errors $(\mathrm{HR}<50$ bpm) are displayed. Although sensors signals were processed on this work, online signal processing may not lead to the same results. This limitation will be overcome with the advance of sensor technologies (measurements and connectivity). 


\section{Conclusion}

This paper presented ARX models for predicting IG during and after PA. We showed that a population-based ARX model may be used for predicting IG 30 min ahead with an acceptable accuracy. We demonstrated that performance of the ARX models increases when the number of training data increases. This result is very interesting, since on T1D patients using an artificial pancreas, the number of quality training data will increase through time. However, patients' physiology also evolves though time, then training data should be correctly chosen. In fact, data collected a long time ago may not be representative of the patient's BG dynamics. Finally, we showed the interest of using meal, insulin, and physical activity information as inputs to increases performance on IG prediction during and after PA. In fact, current models found in the literature are limited to predicting IG during PA (insulin or meal are not used as inputs), or out of a PA (EE is not used as input). The fact of considering the 3 variables as inputs, allows the proposed model to perform accurate IG predictions during and out of PA.

\section{Acknowledgment}

The present study was supported by grants from the French National Agency ANR TECSAN 2015 (DIABELOOP_AP project).

\section{References}

[1] Satish Garg, Ronald L. Brazg, Timothy S. Bailey, Bruce A. Buckingham, Robert H. Slover, David C. Klonoff, John Shin, John B. Welsh, and Francine R. Kaufman. Reduction in duration of hypoglycemia by automatic suspension of insulin delivery: The in-clinic aspire study. $D i$ abetes Technology \& Therapeutics, 14(3):205-209, 2012.

[2] Matthew Stenerson, Fraser Cameron, Darrell M. Wilson, Breanne Harris, Shelby Payne, B. Wayne Bequette, and Bruce A. Buckingham. The impact of accelerometer and heart rate data on hypoglycemia mitigation in type 1 diabetes. Journal of Diabetes Science and Technology, 8(1):64-69, January 2014.

[3] Roman Hovorka, Valentina Canonico, Ludovic J Chassin, Ulrich Haueter, Massimo Massi-Benedetti, Marco Orsini Federici, Thomas R 
Pieber, Helga C Schaller, Lukas Schaupp, Thomas Vering, and Malgorzata E Wilinska. Nonlinear model predictive control of glucose concentration in subjects with type 1 diabetes. Physiological Measurement, 25:905-920, 2004 .

[4] Simone Del Favero, Daniela Bruttomesso, Federico Di Palma, Giordano Lanzola, Roberto Visentin, Alessio Filippi, Rachele Scotton, Chiara Toffanin, Mirko Messori, Stefania Scarpellini, Patrick Keith-Hynes, Boris P. Kovatchev, J. Hans DeVries, Eric Renard, Lalo Magni, Angelo Avogaro, and Claudio Cobelli. First use of model predictive control in outpatient wearable artificial pancreas. Diabetes Care, 37(5):1212-1215, 2014.

[5] Pierre Yves Benhamou, Erik Huneker, Sylvia Franc, Maeva Doron, Guillaume Charpentier, and on behalf of the Diabeloop Consortium. Customization of home closed-loop insulin delivery in adult patients with type 1 diabetes, assisted with structured remote monitoring: the pilot wp7 diabeloop study. Acta Diabetologica, 55(6):549-556, Jun 2018.

[6] Pierre-Yves Benhamou, Sylvia Franc, Yves Reznik, Charles Thivolet, Pauline Schaepelynck, Eric Renard, Bruno Guerci, Lucy Chaillous, Celine Lukas-Croisier, Nathalie Jeandidier, Helene Hanaire, Sophie Borot, Maeva Doron, Pierre Jallon, Ilham Xhaard, Vincent Melki, Laurent Meyer, Brigitte Delemer, Marie Guillouche, Laurene Schoumacker-Ley, Anne Farret, Denis Raccah, Sandrine Lablanche, Michael Joubert, Alfred Penfornis, and Guillaume Charpentier. Closed-loop insulin delivery in adults with type 1 diabetes in real-life conditions: a 12-week multicentre, open-label randomised controlled crossover trial. Lancet Digital Health, 1(1):e17-e25, 2019.

[7] Eray Kulcu, Janet A. Tamada, Gerard Reach, Russell O. Potts, and Matthew J. Lesho. Physiological differences between interstitial glucose and blood glucose measured in human subjects. Diabetes Care, 26(8):2405-2409, 2003.

[8] Elena Daskalaki, Aikaterini Prountzou, Peter Diem, and Stavroula G. Mougiakakou. Real-time adaptive models for the personalized prediction of glycemic profile in type 1 diabetes patients. Diabetes Technology 86 Therapeutics, 14:1520-9156, 2012. 
[9] Marzia Cescon and Rolf Johansson. Glycemic trend prediction using empirical model identification. In Joint 48th IEEE Conference on Decision and Control and 28th Chinese Control Conference, pages 3501-3506. IEEE, 2009.

[10] Marzia Cescon, Rolf Johansson, and Eric Renard. Individualized empirical models of carbohydrate and insulin effects on T1DM blood glucose dynamics. In 2013 IEEE International Conference on Control Applications (CCA) Part of 2013 IEEE Multi-Conference on Systems and Control, pages 258-263. IEEE, 2013.

[11] Marzia Cescon, Rolf Johansson, and Eric Renard. Low-complexity miso models of t1dm glucose metabolism. In 2013 9th Asian Control Conference $(A S C C)$, pages 1-6, June 2013.

[12] Fredrik Stähl and Rolf Johansson. Observer based plasma glucose prediction in type i diabetes. In 2010 IEEE International Conference on Control Applications, pages 1620-1625, Sept 2010.

[13] Meriyan Eren-Oruklu, Ali Cinar, Lauretta Quinn, and Donald Smith. Adaptive control strategy for regulation of blood glucose levels in patients with type 1 diabetes. Journal of Process Control, 19(8):1333 1346, 2009. Special Section on Hybrid Systems: Modeling, Simulation and Optimization.

[14] Dimitri Boiroux, Anne Katrine Duun-Henriksen, Signe Schmidt, Kirsten Nrgaard, Sten Madsbad, Niels Kjlstad Poulsen, Henrik Madsen, and John Bagterp Jrgensen. Overnight glucose control in people with type 1 diabetes. Biomedical Signal Processing and Control, 39:503 - 512, 2018.

[15] Ravi Gondhalekar, Eyal Dassau, and Francis J. Doyle. Periodic zoneMPC with asymmetric costs for outpatient-ready safety of an artificial pancreas to treat type 1 diabetes. Automatica, 71:237 - 246, 2016.

[16] Michael C. Riddell, Dessi P. Zaharieva, Loren Yavelberg, Ali Cinar, and Veronica Jamnik. Exercise and the development of the artificial pancreas: One of the more difficult series of hurdles. Journal of Diabetes Science and Technology, 9(6):1217 - 1226, 2015.

[17] Ravi Reddy, Amanda Wittenberg, Jessica R. Castle, Joseph El Youssef, Kerri Winters-Stone, Melanie Gillingham, and Peter G. Jacobs. Effect 
of aerobic and resistance exercise on glycemic control in adults with type 1 diabetes. Canadian Journal of Diabetes, 2018.

[18] Dimitri Boiroux, John Bagterp Jrgensen, Stephen D. Patek, and Marc D. Breton. The contribution of physical activity in blood glucose concentration for people with type 1 diabetes. IFAC-PapersOnLine, 51(27):270 - 275, 2018. 10th IFAC Symposium on Biological and Medical Systems BMS 2018.

[19] Harry .D Patton, Albert F. Fuchs, Bertil Hille, Allen M. Scher, and Robert Steiner. Circulation, Respiration, Body Fluids, Metabolism and Endocrinology (21st ed). Saunders Company, Philadelphia, PA, 1989.

[20] Martín Hernández-Ordo nez and Daniel Ulises Campos-Delgado. An extension to the compartmental model of type 1 diabetic patients to reproduce exercise periods with glycogen depletion and replenishment. Journal of Biomechanics, 41(4):744 - 752, 2008.

[21] Mohammed Derouich and Abdesslam Boutayeb. The effect of physical exercise on the dynamics of glucose and insulin. Journal of Biomechanics, 35(7):911 - 917, 2002.

[22] Michele Schiavon, Ling Hinshaw, Ashwini Mallad, Chiara Dalla Man, Giovanni Sparacino, Matthew Johnson, Rickey Carter, Rita Basu, Yogish Kudva, Claudio Cobelli, and Ananda Basu. Postprandial glucose fluxes and insulin sensitivity during exercise: A study in healthy individuals. American Journal of Physiology - Endocrinology and Metabolism, 305(4):E557-E566, 2013.

[23] Chiara Dalla Man, Marc D. Breton, and Claudio Cobelli. Physical activity into the meal glucoseinsulin model of type 1 diabetes: In silico studies. Journal of Diabetes Science and Technology, 3(1):56-67, 2009. PMID: 20046650.

[24] M. Cescon and E. Renard. Adaptive subspace-based prediction of T1DM glycemia. In 50th IEEE Conference on Decision and Control and European Control Conference, page 51645169. IEEE, 2011.

[25] Per Hildebrandt, Jesper Mehlsen, Leif Sestoft, and Steen Levin Nielsen. Mild mental stress in diabetes: changes in heart rate and subcutaneous blood-flow. Clinical Physiology, 5(4):371-376, 1985. 
[26] Isuru S. Dasanayake, Dale E. Seborg, Jordan E. Pinsker, Francis J. Doyle, and Eyal Dassau. Empirical dynamic model identification for blood-glucose dynamics in response to physical activity. In 201554 th IEEE Conference on Decision and Control (CDC), pages 3834-3839, Dec 2015.

[27] Naviyn Prabhu Balakrishnan, Lakshminarayanan Samavedham, and Gade Pandu Rangaiah. Personalized hybrid models for exercise, meal, and insulin interventions in type 1 diabetic children and adolescents. Industrial \& Engineering Chemistry Research, 52(36):13020-13033, 2013.

[28] Kamuran Turksoy, Elif S. Bayrak, Lauretta Quinn, Elizabeth Littlejohn, and Ali Cinar. Multivariable adaptive closed-loop control of an artificial pancreas without meal and activity announcement. Diabetes Technology ES Therapeutics, 15(5):386-400, 2013.

[29] Hector M Romero-Ugalde, M Garnotel, M Doron, P Jallon, G Charpentier, S Franc, E Huneker, C Simon, and S Bonnet. An original piecewise model for computing energy expenditure from accelerometer and heart rate signals. Physiological Measurement, 38(8):1599, 2017.

[30] Kamuran Turksoy, Iman Hajizadeh, Nicole Hobbs, Jennifer M. Kilkus, Elizabeth Littlejohn, Sediqeh Samadi, Jianyuan Feng, Mert Sevil, Caterina Lazaro, Julia Ritthaler, Brooks A Hibner, Nancy A. Devine, Lauretta Quinn, and Ali Cinar. Multivariable artificial pancreas for various exercise types and intensities. Diabetes technology $\mathcal{E}_{3}$ therapeutics, 20(10), 2018.

[31] Amin Soltanieh and Oluwaseyi Ogun. Identification of nonlinear multi input multi output model of PEM fuel cell stack system. In Electrical Engineering (ICEE), Iranian Conference on, pages 887-892, May 2018.

[32] Shahnaz TayebiHaghighi Farzin Piltan, Nasri B. Sulaiman, and P Wouters. Comparative study between ARX and ARMAX system identification. I.J. Intelligent Systems and Applications, 2:25-34, 2017.

[33] Brian M. Sandroff, Barry J. Riskin, Stamatis Agiovlasitis, and Robert W. Motl. Accelerometer cut-points derived during over-ground walking in persons with mild, moderate, and severe multiple sclerosis. Journal of the Neurological Sciences, 340(1):50 - 57, 2014. 
[34] Ling Hinshaw, Chiara Dalla Man, Debashis K. Nandy, Ahmed Saad, Adil E. Bharucha, James A. Levine, Robert A. Rizza, Rita Basu, Rickey E. Carter, Claudio Cobelli, Yogish C. Kudva, and Ananda Basu. Diurnal pattern of insulin action in type 1 diabetes. Diabetes, 62(7):2223-2229, 2013.

[35] American Diabetes Association. Postprandial blood glucose. Diabetes Care, 24(4):775-778, 2001.

[36] Tom Van Herpe, Marcelo Espinoza, Bert Pluymers, Ivan Goethals, Pieter Wouters, Greet Van den Berghe, and Bart De Moor. An adaptive input-output modeling approach for predicting the glycemia of critically ill patients. Physiological Measurement, 27(11):1057, 2006.

[37] Thomas W. MacFarland and Jan M. Yates. Wilcoxon Matched-Pairs Signed-Ranks Test, pages 133-175. Springer International Publishing, Cham, 2016. 